\title{
REVIEW
}

Open Access

\section{Effects of reconstruction techniques after proximal gastrectomy: a systematic review and meta-analysis}

Zakari Shaibu ${ }^{1,2} \mathbb{D}$, Zhihong Chen ${ }^{1 *}$, Said Abdulrahman Salim Mzee ${ }^{2,3} \mathbb{E}$, Acquah Theophilus $^{1,2}$ and Isah Adamu Danbala ${ }^{2,3}$

\begin{abstract}
Background: Additional studies comparing several reconstruction methods after proximal gastrectomy have been published; of note, it is necessary to update systematic reviews and meta-analysis from the current evidence-based literature.

Aim: To expand the current knowledge on feasibility and safety, and also to analyze postoperative outcomes of several reconstructive techniques after proximal gastrectomy.

Methods: PubMed, Google Scholar, and Medline databases were searched for original studies, and relevant literature published between the years 1966 and 2019 concerning various reconstructive techniques on proximal gastrectomy were selected. The postoperative outcomes and complications of the reconstructive techniques were assessed. Meta-analyses were performed using Rev-Man 5.0. A total of 29 studies investigating postoperative outcomes of double tract reconstruction, jejunal pouch interposition, jejunal interposition, esophagogastrostomy, and double flap reconstruction were finally selected in the quantitative analysis.

Result: Pooled incidences of reflux esophagitis for double tract reconstruction, jejunal pouch interposition, jejunal interposition esophagogastrostomy, and double flap reconstruction were 8.6\%, 13.8\%, 13.8\%, 19.3\%, and 8.9\% respectively. Meta-analysis showed a decreased length of hospital in the JI group as compared to the JPI group (heterogeneity: $\mathrm{Chi}^{2}=1.34 \mathrm{df}=1(P=0.25) ; P^{2}=26 \%$, test for overall effect: $Z=2.22(P=0.03)$. There was also a significant difference between $\mathrm{J}$ and $\mathrm{EG}$ in length of hospital stay with heterogeneity: $\mathrm{Chi}^{2}=1.40, \mathrm{df}=3(P=0.71)$; $P^{2}=0 \%$, test for overall effect: $Z=5.04(P<0.00001)$. Operative time was less in the EG group as compared to the group (heterogeneity: $\mathrm{Chi}^{2}=31.09, \mathrm{df}=5(P<0.00001) ; P^{2}=84 \%$, test for overall effect: $Z=32.35(P<0.00001)$.

(Continued on next page)
\end{abstract}

\footnotetext{
* Correspondence: chenzhi-hong@163.com

'Department of Gastrointestinal Surgery, Affiliated People's Hospital of Jiangsu University, Zhenjiang 212002, Jiangsu, People's Republic of China

Full list of author information is available at the end of the article
}

C C The Author(s). 2020 Open Access This article is licensed under a Creative Commons Attribution 4.0 International License, which permits use, sharing, adaptation, distribution and reproduction in any medium or format, as long as you give appropriate credit to the original author(s) and the source, provide a link to the Creative Commons licence, and indicate if changes were made. The images or other third party material in this article are included in the article's Creative Commons licence, unless indicated otherwise in a credit line to the material. If material is not included in the article's Creative Commons licence and your intended use is not permitted by statutory regulation or exceeds the permitted use, you will need to obtain permission directly from the copyright holder. To view a copy of this licence, visit http://creativecommons.org/licenses/by/4.0/. The Creative Commons Public Domain Dedication waiver (http://creativecommons.org/publicdomain/zero/1.0/) applies to the data made available in this article, unless otherwise stated in a credit line to the data. 


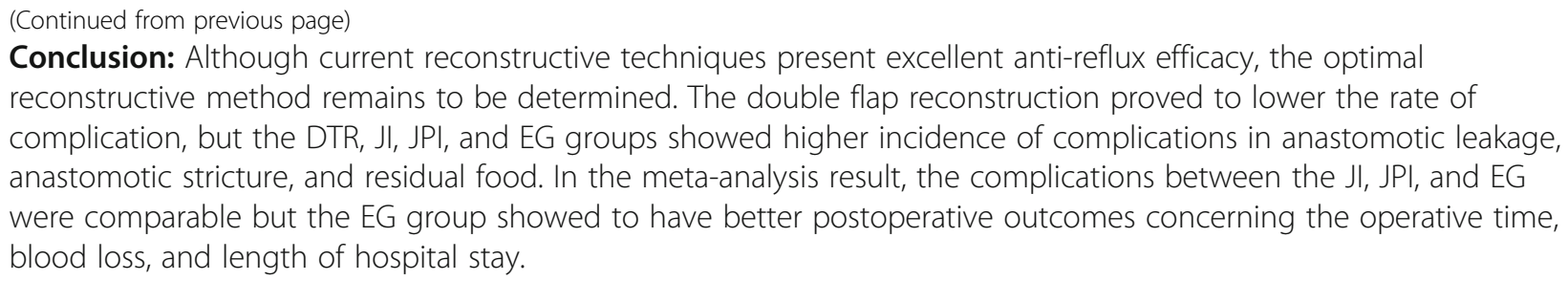

Keywords: Proximal gastrectomy, Double tract reconstruction, Jejunal pouch interposition, Jejunal interposition, Esophagogastrostomy, Double flap, Gastric cancer or neoplasms

\section{Background}

Gastric malignancy is one of the established malignancies associated with high incidence of mortality worldwide. Recent articles have reported the modern diagnostic and treatment methods on eradication of Helicobacter pylori to have a direct link on the decline in gastric cancer [1-3]. Gastric cancer rates have dwindled in diagnosis across the world but lamentably, it remains to be a conundrum in the surgical field $[4,5]$. The incidence of upper-third gastric cancer, including early gastric cancers, is increasing in Korea, China, and Japan [6-8]. And therefore, cancer-related death rate for proximal gastric cancer (PGC) is higher compared to cancers at other sites of the stomach [9-11]. Several methods tested the sole therapeutic agent deemed reliable as a medicinal procedure of gastric cancer remains to be surgical resection. Despite the fact that there is no general agreement on a preference of procedures as a remedy for PGC [12], additionally, there is still no consensus on the choice of surgical procedures for PGC.

Two distinct stomach resections by a surgical procedure for proximity gastric cancer include total gastrectomy (TG) and proximal gastrectomy (PG). The type of gastrectomy that is chosen of the two by GI surgeons usually relies upon the tumor's size, stage, and volume of the remnant in the stomach [13]. Accordingly, various types of reconstructions have been investigated. Japanese gastric cancer treatment guidelines propose 3 types of reconstructions for proximal gastrectomy: esophagogastrostomy, jejunal interposition, and double tract jejunal interposition [14]. Furthermore, jejunal pouch interposition and gastric tube stomach esophagogastrostomy are still perceived as dependable methods [15].

Although total gastrectomy may grant more extended lymph node (LN) dissection, proximal gastrectomy is effective as it is able to provide comparable recurrence and survival rates without compromising the physiological functions of the gastric remnant [9]. Actually, it has been expressed by several authors that proximal gastrectomy is the best appropriate surgical operation of early cancer occurring in the upper part of the stomach, since it has the best durability and safety for patients.
Their findings have concluded that, evidence as stated prior, proximal gastrectomy has the same survival rates of patients who received the procedure compared to those who received total gastrectomy, while maintaining the physiologic functions of gastric remnant [16]. Nonetheless, proximal gastrectomy may have its eminence in gastric cancer resections; it also comes with undesirable outcomes as well. There is a high primary concern of its possible postoperative complications which consist of reflux esophagitis, which causes excessive heartburn, chest pain, regurgitation of sourness, and anorexia. These complications can diminish the postoperative quality of lives in gastrectomy patients [17].

It should be noted that multiple methods are possible for reconstruction after proximal gastrectomy. Although other additional reconstruction procedures, such as jejunal interposition (JI) [16], jejunal pouch interposition (JPI) [18], and the double-tract reconstruction (DTR) method, [19] in which some distance is maintained between the esophagus and gastric remnant, are efficient in preventing reflux to some extent, the downside is that these procedures can cause other symptoms that are uncommon with EG, such as obstruction and difficulty in endoscopic surveillance of the gastric remnant after surgery [20]. Alternatively, due to the high presence of reflux esophagitis after simple esophagogastrostomy, it has inspired the advancement of new techniques for reconstruction that purposefully aids to prevent reflux, two of which incorporate jejunal pouch interposition and jejunal interposition $[16,21]$. The most common type of reconstruction is esophagogastrostomy. A questionnaire was done by 145 Japanese institutions, and it indicated that esophagogastrostomy is desired after a proximal gastrectomy by approximately $50 \%$ of institutions [17]. Kamikawa procedure also known as double flap technique (DFT), which was first reported in 1998, is an anti-reflux procedure during EG after PG. DFT consists of a unique multistep process involving the creation of an $\mathrm{H}$-shaped seromuscular double-flap, fixing the esophagus, and the gastric remnant, as well as anastomosis and closure of the double-flap, all of which are basically carried out by hand-sewn techniques. During this 
procedure, the distal esophagus and anastomosis are embedded in the submucosal layer of the gastric remnant and covered by the seromuscular double-flap, which is meant to function as a one-way valve to stop reflux [22].

The purpose of this study was to disclose the postoperative outcomes of different reconstruction techniques following a proximal gastrectomy.

\section{Materials and method}

Extraction was independently performed by using specially designed data extraction sheets. After we collected 29 full papers, authors, nationality, study design, publication year, type of surgery, and the number of patients were shown in Table 1. Operation time, blood loss, hospital length of stay, anastomotic leakage, anastomotic stricture, reflux esophagitis, and residual food were all considered as the postoperative outcomes.

\section{Data extraction}

General characteristics of included studies, such as the country, study design, type of reconstruction, publication year, and a number of patients, were shown in Table 1 . The reconstruction types were classified into 5 groups: double tract reconstruction, jejunal pouch interposition, jejunal interposition, esophagogastrostomy, and double flap technique. Incidence of postoperative outcomes such as anastomotic strictures, anastomotic leakage, residual food, and reflux esophagitis was confirmed by endoscopic examination. Reflux esophagitis was classified by the Los Angeles classification; degree B or more severe degrees were extracted. In studies reporting incidences of reflux esophagitis during various periods, incidences during the 12th month were considered.

Studies separately reporting each postoperative complication were also selected where the most frequently

Table 1 Basic characteristics of included studies

\begin{tabular}{|c|c|c|c|c|c|}
\hline Authors & Nationality & year & Design & Groups & Number of patients \\
\hline wright et al. [23] & Scotland & 1987 & RS & J & 30 \\
\hline Kameyama et al. [19] & Japan & 2004 & $\mathrm{RC}$ & $\mathrm{Jl} / \mathrm{JPI}$ & $13 / 59$ \\
\hline Senmaru et al. [24] & Japan & 1999 & $\mathrm{RC}$ & $\mathrm{Jl} / \mathrm{JPI}$ & $12 / 12$ \\
\hline Isobe et al. [25] & Japan & 2014 & $\mathrm{RC}$ & EG/JI/JPI & $66 / 23 / 12$ \\
\hline kazuhiro et al. [26] & Japan & 1998 & $\mathrm{RC}$ & EG/J & $11 / 14$ \\
\hline Adachi et al. [27] & Japan & 1999 & $\mathrm{RC}$ & ال & $16 / 14$ \\
\hline kondoh et al. [28] & Japan & 2006 & RS & EG & 10 \\
\hline Yasuda et al. [29] & Japan & 2015 & $\mathrm{RC}$ & EG/J & $25 / 21$ \\
\hline zhao et al. [21] & China & 2014 & RS & ال & 35 \\
\hline Ahn et al. [30] & Japan & 2014 & RS & DTR & 43 \\
\hline Kim et al. [31] & Korea & 2016 & RS & DTR & 27 \\
\hline Zhang et al. [22] & China & 2018 & RS & EG & 62 \\
\hline Kamitaka et al. [32] & Japan & 2017 & RS & DTR & 10 \\
\hline Nomura et al. [17] & Japan & 2019 & PS & DTR/JI & $15 / 15$ \\
\hline Sugiyama et al. [33] & Japan & 2018 & RS & DTR & 10 \\
\hline Tokunaga et al. [34] & Japan & 2008 & RS & EG/J & $49 / 58$ \\
\hline Ohashi et al. [35] & Japan & 2015 & RS & ال & 65 \\
\hline Tanaka et al. [32] & Japan & 2017 & RS & DTR & 10 \\
\hline Aburatani et al. [36] & Japan & 2017 & RS & DTR/EG & $19 / 22$ \\
\hline Yang et al. [37] & Korea & 2016 & RS & DTR & 16 \\
\hline Nakmura et al. [38] & Japan & 2014 & RS & EG/JI/JPI & $65 / 25 / 12$ \\
\hline Hong et al. [39] & China & 2015 & RS & DTR & 21 \\
\hline Katai et al. [14] & Japan & 2010 & RS & ال & 128 \\
\hline Masuzawa [40]. & Japan & 2013 & RS & EG/J & $49 / 32$ \\
\hline Takayama [41]. & Japan & 2018 & RS & 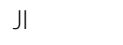 & 32 \\
\hline Kano et al. [42] & Japan & 2019 & RS & DFR & 51 \\
\hline Koruda et al. [20] & Japan & 2019 & RS & DFR & 464 \\
\hline Omori et al. [43] & Japan & 2017 & RS & DFR & 32 \\
\hline Saeki et al. [44] & Japan & 2018 & RS & DFR & 13 \\
\hline
\end{tabular}


observed complication was considered. All analysis was based on previously published studies, meaning, no ethical approval and patient consent were required.

\section{Characteristics of included studies}

The characteristics of the 29 studies are listed in Table 1. The published year ranged from 1966 to 2019. The studies included 21 retrospective studies, 1 prospective studies, and 6 randomized control. The reconstruction types were classified into 5 groups: double tract reconstruction, jejunal pouch interposition, jejunal interposition, esophagogastrostomy, and double flap technique (Fig. 15 a-e).

\section{Inclusion criteria}

- Only full published paper in English or translated paper.

- Comparative and non-comparative studies included.

- Open or laparoscopic procedure of proximal gastrectomy.

- Laparoscopic or open techniques

\section{Exclusion criteria}

- Animals or lab studies excluded.

- Case reports, comments, letters, and reviews without original date excluded.

- Postoperative outcomes are not recorded.

- Total gastrectomy, subtotal gastrectomy, and distal gastrectomy are excluded.

\section{Statistical analysis}

Statistical analysis was performed using the Review Manager (RevMan) software, version 5.0 offered by the Cochrane collaboration. Continuous variables were pooled using the mean difference (MD) with a 95\% confidence interval (95\% CI), and dichotomous variables were pooled using the odds ratio (OR) with a 95\% CI. Random effect and fixed effect models were computed under statistical methods of Mantel-Haenszel (for OR or RR). Heterogeneity among studies was assessed using the inconsistency statistic $(I)$. If $I$ was $<50 \%$, the eligible studies were considered to be homogenous; hence, the fixed effect model was used. In contrast, if $I$ was $>50 \%$, the pooled results were said to be significant, heterogeneous, and the random effect model was used instead.

\section{Results}

General characteristic of the analyzed patients

A total of 720 studies were searched. Of these searched studies, 420 remaining after duplicates were removed, and 420 articles were screened and resulted in 314 irrelevant, 27 reports, 19 meta-analyses, 17 reviews and comment, and 7 animal studies. A total of 36 full-text articles were carefully studied and 7 studies lacking targeted data. The remaining 29 studies were included as shown in Fig. 1. Of the 29 studies, 29 were analyzed as quantitative studies and 8 out of the 29 for qualitative studies. The 29 studies included in quantitative studies comprised of several reconstructive techniques. Their studies were 3 papers from china, Zhao et al. [39], Zhang et al. [24], and Hong et al. [25], 23 papers from Japan,

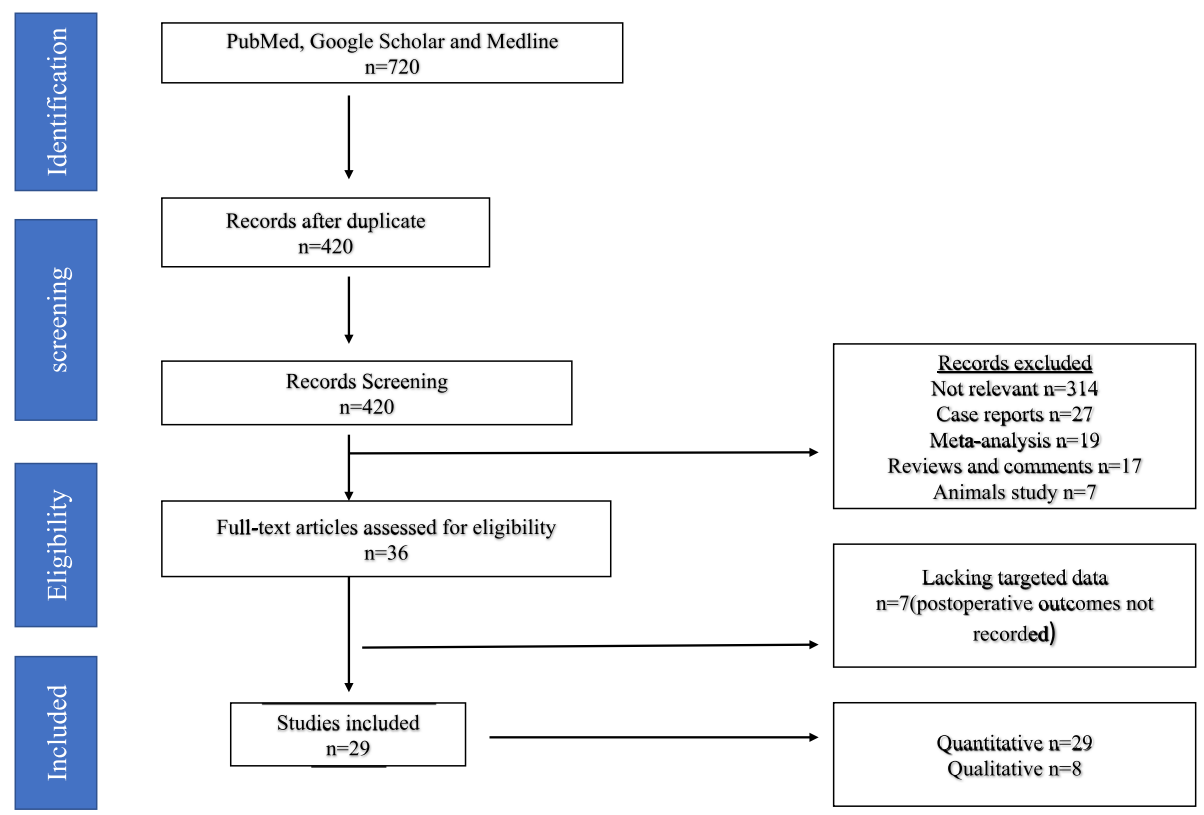

Fig. 1 Flow chart of the literature screening, exclusion and inclusion process 
Table 2 Qualitative analysis postoperative outcomes

\begin{tabular}{|c|c|c|c|c|}
\hline Authors & Reflux esophagitis & Anastomotic stricture & Anastomotic leakage & Residual food \\
\hline \multicolumn{5}{|l|}{ Double tract } \\
\hline Ahn et al. [30] & $2 / 43(4.65 \%)$ & $2 / 43(4.65 \%)$ & - & $21 / 43(48.9 \%)$ \\
\hline Kim et al. [31] & $2 / 17(11.8 \%)$ & 0/17 (0\%) & $1 / 17(5.9 \%)$ & - \\
\hline Kamitaka et al. [32] & $2 / 10(20 \%)$ & - & 0/10 (0\%) & - \\
\hline Nomura et al. [17] & $1 / 15(6.7 \%)$ & $1 / 15(3.3 \%)$ & 0/15 (0\%) & $2 / 15(13.3 \%)$ \\
\hline Sugiyama et al. [33] & - & 0/10 (0\%) & $1 / 10(10 \%)$ & - \\
\hline Aburatani et al. [47] & $2 / 19(10.5 \%)$ & 0 & 0 & - \\
\hline Yang et al. [37] & 0 & - & - & - \\
\hline Tanaka et al. [32] & $2 / 10(20 \%)$ & 0 & 0 & 0 \\
\hline Hong et al. [39] & 0 & 0 & 0 & - \\
\hline Total & $11 / 114(9.6 \%)$ & $3 / 85(3.5 \%)$ & $2 / 52(3.9 \%)$ & 23/58 (39.6\%) \\
\hline \multicolumn{5}{|l|}{ Jejunal pouch interposition } \\
\hline Senmaru et al. [24] & - & $1 / 12(8.3 \%)$ & $0(0 \%)$ & - \\
\hline Kameyama et al. [19] & $6 / 46(13.0 \%)$ & - & 9/46 (15.3\%) & $23 / 46(50.0 \%)$ \\
\hline Isobe et al. [25] & $2 / 12(16.7)$ & $1 / 12(8.3 \%)$ & $1 / 12(8.3 \%)$ & - \\
\hline Nakamura et al [38]. & - & $1 / 12(8.3 \%)$ & 0 & $11 / 12(91.7 \%)$ \\
\hline Total & $8 / 58(13.8 \%)$ & $3 / 36(8.3 \%)$ & 10/58 (17.2\%) & $34 / 58(58.6 \%)$ \\
\hline \multicolumn{5}{|l|}{ Jejunal interposition } \\
\hline Wright et al. [23] & $2 / 30(6.7 \%)$ & $1 / 30(3.3 \%)$ & $3 / 30(10 \%)$ & $8 / 30(26.7 \%)$ \\
\hline Senmaru et al. [24] & - & $2 / 12(16.7 \%)$ & $1 / 12(8.3 \%)$ & - \\
\hline Kameyama et al. [19] & $3 / 10(30.0 \%)$ & - & $0 / 10(0.0 \%)$ & $3 / 10(30.0 \%)$ \\
\hline Isobe et al. [25] & $3 / 23(13.0)$ & 0/23 (0\%) & $3 / 23(13.0)$ & - \\
\hline Kazuhiro et al. [26] & 0/14 (0\%) & 9/14 (64.3\%) & 0/14 (0\%) & - \\
\hline Adachi et al. [27] & 0/16 (0\%) & $1 / 16(6.3 \%)$ & $0 / 16(0 \%)$ & - \\
\hline Yasuda et al. [29] & $1 / 23(5 \%)$ & $3 / 21(14.3 \%)$ & $2 / 21(10 \%)$ & $17 / 17(100 \%)$ \\
\hline Zhao et al. [21] & $2 / 31(6.5 \%)$ & - & 0/35 (0\%) & - \\
\hline Nomura et al. [17] & $1 / 15(6.7 \%)$ & $1 / 15(3.3 \%)$ & 0/15 (0\%) & 4/15 (26.7\%) \\
\hline Tokunaga et al. [46] & $3 / 45(7 \%)$ & - & - & - \\
\hline Nakamura et al. [38] & - & $7 / 22(31.8 \%)$ & $1 / 25(4 \%)$ & 7/22 (31.8\%) \\
\hline Takayama et al. [41] & - & $1 / 32(3.1 \%)$ & - & - \\
\hline Ohashi et al. [35] & 22/65 (34\%) & - & 6/65 (9\%) & - \\
\hline Katai et al. [48] & - & 13/128 (10.2\%) & $1 / 128(0.8 \%)$ & - \\
\hline Masuzawa et al. [40] & $5 / 32(15.6 \%)$ & $1 / 32(3.1 \%)$ & 0 & - \\
\hline Total & $42 / 304(13.8 \%)$ & $39 / 345(11.3 \%)$ & $15 / 369$ (4.1\%) & 39/94 (41.5\%) \\
\hline \multicolumn{5}{|l|}{ Esophagogastrostomy } \\
\hline Isobe et al. [25] & $12 / 66(18.2 \%)$ & $2 / 66(3.0 \%)$ & $1 / 66(1.5 \%)$ & - \\
\hline Kazuhiro et al. [26] & 4/10 (40\%) & $2 / 10(20 \%)$ & $2 / 11(18.2 \%)$ & - \\
\hline Kondoh et al. [28] & $4 / 10(40 \%)$ & $4 / 10(40 \%)$ & 0/10 (0\%) & - \\
\hline Yasuda et al. [29] & $1 / 23(4.3 \%)$ & $0 / 25(0 \%)$ & 0/25 (0\%) & - \\
\hline Zhang et al. [22] & 9/62 (14.5\%) & $11 / 62(7.1 \%)$ & $5 / 62(8.1 \%)$ & - \\
\hline Tokunaga et al. [46] & $3 / 38(8 \%)$ & - & - & - \\
\hline Nakamura et al. [38] & - & 12/55 (21.8\%) & 0 & $12 / 55(21.8 \%)$ \\
\hline Masuzawa et al. [40] & 9/49 (18.4\%) & $2 / 49(4.1 \%)$ & 0 & \\
\hline Abutarani et al. [47] & $12 / 22(54.5 \%)$ & $6 / 22(27.3 \%)$ & 0 & \\
\hline
\end{tabular}


Table 2 Qualitative analysis postoperative outcomes (Continued)

\begin{tabular}{|c|c|c|c|c|}
\hline Authors & Reflux esophagitis & Anastomotic stricture & Anastomotic leakage & Residual food \\
\hline Total & $54 / 280(19.3 \%)$ & 39/299 (13.0\%) & $8 / 174(4.6 \%)$ & $12 / 55(21.8 \%)$ \\
\hline \multicolumn{5}{|c|}{ Double flap reconstruction } \\
\hline Kano et al. [42] & 3/51 (5.9\%) & $4 / 51(8 \%)$ & 0/51 (0\%) & 2/51 (3.9\%) \\
\hline Koruda et al. [20] & $46 / 464(10.6 \%)$ & 26/464 (5.5\%) & $7 / 464(1.5 \%)$ & - \\
\hline Omori et al. [43] & $0 / 32(0 \%)$ & 0/32 (0\%) & 0/32 (0\%) & - \\
\hline Saeki et al. [44] & $1 / 13(7.7 \%)$ & - & $1 / 13(7.7 \%)$ & - \\
\hline Total & $50 / 560(8.9 \%)$ & $30 / 547$ (5.5\%) & $8 / 560(1.4 \%)$ & $2 / 51(3.9 \%)$ \\
\hline
\end{tabular}

senmaru et al. [26], kameyama et al. [21], isobe et al. [27], kazuhiro et al. [28], adachi et al. [29], kondoh et al. [30], yasuda et al. [32], ahn et al. [45], nomura et al. [33], sugiyama et al. [46], tokunaga et al. [35], ohashi et al. [47], tanaka et al. [38], aburatani et al .[40], nakamura et al. [41], katai et al. [16], masuzawa et al. [42] and takayama et al. [43], kano et al. [44], koruda et al. [22], omori et al [31], and saeki et al. [37], 2 papers from Korea, Kim et al. [23] and yang et al. [34], and 1 paper from Scotland, wright et al. [36]. Studies reported postoperative outcomes such as reflux esophagitis, anastomotic leakage, anastomotic stricture, and residual food shown in Table 2.

The qualitative studies included 8 studies comparing JI VS JPI and JI VS EG after proximal gastrectomy. All the studies included are from japan: Yasuda et al. [32], kameyama et al. [21], isobe et al. [27], and Kazuhiro et al. [28], tokunaga et al., nakamura et al., masuzawa et al., and Senmaru et al. [26]. Studies for analysis included operative time, blood loss and hospital length of stay, reflux esophagitis, anastomotic stricture, and anastomotic leakage as shown in Table 3.

\section{Results of qualitative studies \\ Double tract reconstruction}

Eight studies [19, 23, 25, 34, 38, 45, 46, 48] reported double tract reconstruction with a total 171 patients involved. Postoperative outcomes such as reflux esophagitis, anastomotic stricture, anastomotic leakage, and residual food were observed having 9.6\%, 3.5\%, 3.9\%, and $39.6 \%$ respectively. Reflux esophagitis and residual food were the most frequently observed as shown in Table 2.

Table 3 Quantitative studies between JI, JPI, and EG

\begin{tabular}{|c|c|c|c|c|c|c|c|c|}
\hline Reference & $\begin{array}{l}\text { Reflux } \\
\text { Esophagitis }\end{array}$ & $\begin{array}{l}\text { Anastomotic } \\
\text { stricture }\end{array}$ & $\begin{array}{l}\text { Anastomotic } \\
\text { leakage }\end{array}$ & Infection & $\begin{array}{l}\text { Residual } \\
\text { food }\end{array}$ & Operation time & Blood loss & LOS \\
\hline \multicolumn{9}{|l|}{ J//JPI } \\
\hline $\begin{array}{l}\text { Senmaru } \\
\text { et al. [24] }\end{array}$ & - & $2 / 1$ & $1 / 0$ & $1 / 0$ & - & $\begin{array}{l}297.8+17.5 / 282.9 \\
+18.6\end{array}$ & - & $\begin{array}{l}38.6+3.5 / \\
43.5+5.9\end{array}$ \\
\hline $\begin{array}{l}\text { Kameyama } \\
\text { et al. [19] }\end{array}$ & $3 / 2$ & - & $0 / 1$ & - & $30.0 \% / 50.0 \%$ & - & - & - \\
\hline $\begin{array}{l}\text { Isobe } \\
\text { et al. [25] }\end{array}$ & $3 / 2$ & $0 / 1$ & $3 / 1$ & - & - & $\begin{array}{l}251.7+43.6 / 270.2 \\
+50.0\end{array}$ & $\begin{array}{l}230.4+204.5 / \\
333.9+354.4\end{array}$ & $\begin{array}{l}24.1+17.7 / \\
21.7+16.3\end{array}$ \\
\hline $\begin{array}{l}\text { Nakamura } \\
\text { et al. [38] }\end{array}$ & $11-$ & $7 / 1$ & $1 / 0$ & - & - & $281+69 / 311+68$ & $\begin{array}{l}393+338 / \\
402+385\end{array}$ & - \\
\hline \multicolumn{9}{|l|}{ J/EG } \\
\hline $\begin{array}{l}\text { Isobe } \\
\text { et al. [25] }\end{array}$ & $3 / 12$ & $2 / 0$ & $3 / 1$ & - & - & $\begin{array}{l}251.7+43.6 / 199.0 \\
+43.1\end{array}$ & $\begin{array}{l}230.4+204.5 / \\
176.5+144.2\end{array}$ & $\begin{array}{l}24.1+17.7 / \\
15.6+10.4\end{array}$ \\
\hline $\begin{array}{l}\text { Kazuhiro } \\
\text { et al. [26] }\end{array}$ & $0 / 4$ & - & $0 / 2$ & $2 / 0$ & - & $\begin{array}{l}241.2+10.2 / 186.9 \\
+11.7\end{array}$ & $\begin{array}{l}424 . .6+64.1 / \\
324.0+61.4\end{array}$ & $\begin{array}{l}35.0+3.9 / \\
28.4+4.3\end{array}$ \\
\hline $\begin{array}{l}\text { Yasuda } \\
\text { et al. [29] }\end{array}$ & $1 / 1$ & $3 / 0$ & $2 / 0$ & $2 / 3$ & - & $\begin{array}{l}268.8+59.6 / 286.4 \\
+54.3\end{array}$ & $\begin{array}{l}307.4+264.8 / \\
299.2+334.5\end{array}$ & $\begin{array}{l}29.4+19.8 / \\
18.6+3.6\end{array}$ \\
\hline $\begin{array}{l}\text { Tokunaga } \\
\text { et al. [34] }\end{array}$ & $3 / 3$ & - & - & - & - & $267+11 / 203+9$ & $\begin{array}{l}287+32 / \\
252+50\end{array}$ & - \\
\hline $\begin{array}{l}\text { Nakamura } \\
\text { et al. [38] }\end{array}$ & $11 / 12$ & $7 / 12$ & $1 / 0$ & - & - & $281+69 / 198+25$ & $\begin{array}{l}393+338 / \\
179+158\end{array}$ & - \\
\hline $\begin{array}{l}\text { Masuzawa } \\
\text { et al. [40] }\end{array}$ & $5 / 9$ & $1 / 2$ & $0 / 0$ & - & - & $230+43 / 185+48$ & $\begin{array}{l}331+182 / \\
280+247\end{array}$ & $\begin{array}{l}23+31 / \\
20+17\end{array}$ \\
\hline
\end{tabular}




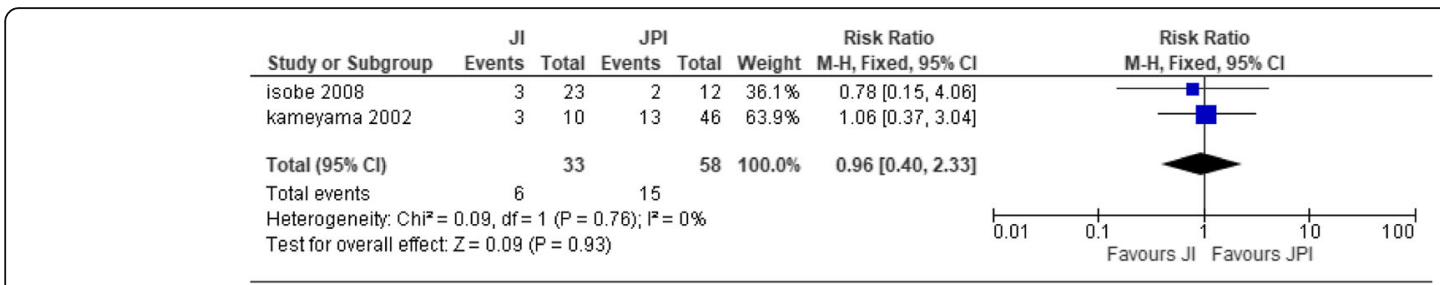

Fig. 2 Forest plot of reflux esophagitis

\section{Jejunal pouch interposition}

A total of four studies [21, 26, 27, 41] concentrated on the postoperative condition of patients who underwent a jejunal pouch interposition with a total of 95 patients involved. Incidences of patients who developed postoperative outcomes such as reflux esophagitis, anastomotic stricture, anastomotic leakage, and residual food were $13.8 \%, 8.3 \%, 17.2 \%$, and $58.6 \%$ respectively. Residual food and anastomotic leakage were the most observed. The incidence of residual food was reported to be high (Table 2).

\section{Jejunal interposition}

A total of fifteen studies $[19,21,26-29,32,35,36$, $39,41-43,47]$ reported postoperative conditions of the jejunal interposition with 519 patients that were involved. Incidence of postoperative outcomes was $13.8 \%, 11.3 \%, 4.1 \%$, and $41.5 \%$ for reflux esophagitis, anastomotic stricture, anastomotic leakage, and residual food respectively. Reflux esophagitis and residual food were the most observed. Residual food had a high incidence (Table 2).

\section{Esophagogastrostomy}

A total of nine studies [24, 27, 28, 30, 32, 35, 41, 42, 48] reported on esophagogastrostomy. Analysis of the results included reflux esophagitis 19.3\%, anastomotic stricture $13.0 \%$, anastomotic leakage $4.6 \%$, and residual food $21.8 \%$. These calculated values show that reflux esophagitis and anastomotic leakage were the most frequently observed postoperative outcomes (Table 2).

\section{Double flap technique}

Four studies [22, 31, 37, 44] reported double flap reconstruction with a total of 560 patients involved. Postoperative results including reflux esophagitis, anastomotic stricture, anastomotic leakage, and residual food were observed with an incidence of $8.9 \%, 5.5 \%, 1.4 \%$, and $3.9 \%$ respectively. The occurrence of reflux esophagitis and anastomotic stricture was the most frequently observed complication (Table 2).

\section{Meta-analysis result for Jl versus JPI Reflux esophagitis}

Two papers [21, 27] were used for the analysis with JI ( $n$ $=33)$ patients and JPI $(n=58)$ patients. The two studies were homogenous; hence, fixed effect was implemented [heterogeneity: $\left.\mathrm{Chi}^{2}=0.09, \mathrm{df}=1(P=0.76) ; I^{2}=0 \%\right]$. There was no statistical significance difference between the two types of reconstructive technique (test for overall effect: $Z=0.09(P=0.93))$ (Fig. 2).

\section{Anastomotic leakage}

Three studies [26, 27, 41] were used for the analysis with JI $(n=55)$ participants and JPI $(n=70)$ participants. The two studies were moderately heterogeneous; therefore, random effect was used [heterogeneity: $\mathrm{Chi}^{2}=4.39$, df $\left.=2(P=0.11) ; \mathrm{I}^{2}=54 \%\right]$. There was no statistical significance difference between JI and JPI (test for overall effect: $\mathrm{Z}=1.46(\mathrm{P}=0.14)$ ) (Fig. 3$)$.

\section{Anastomotic stricture}

Four papers [21, 26, 27, 41] were used for the analysis of JI $(n=70)$ patients and JPI $(n=136)$ patients. Two

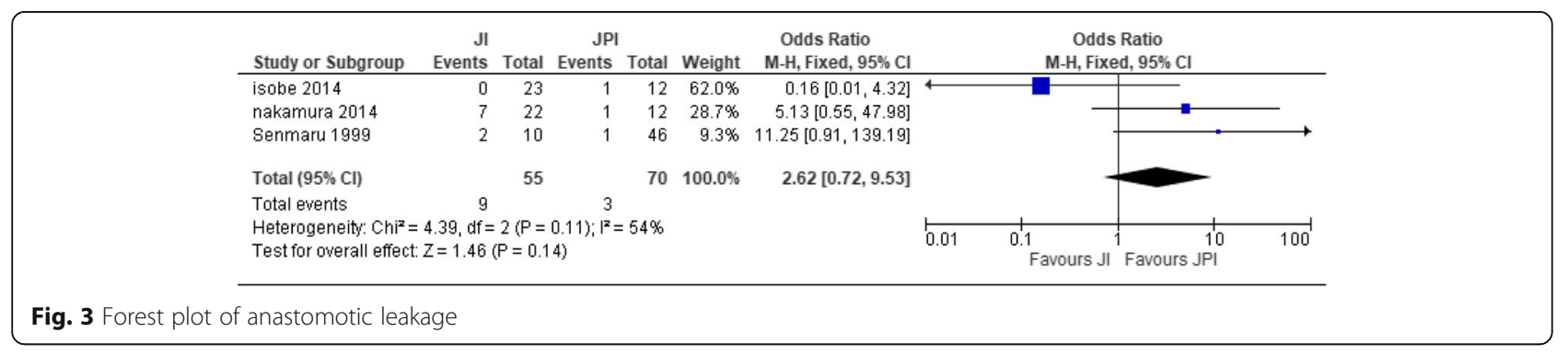




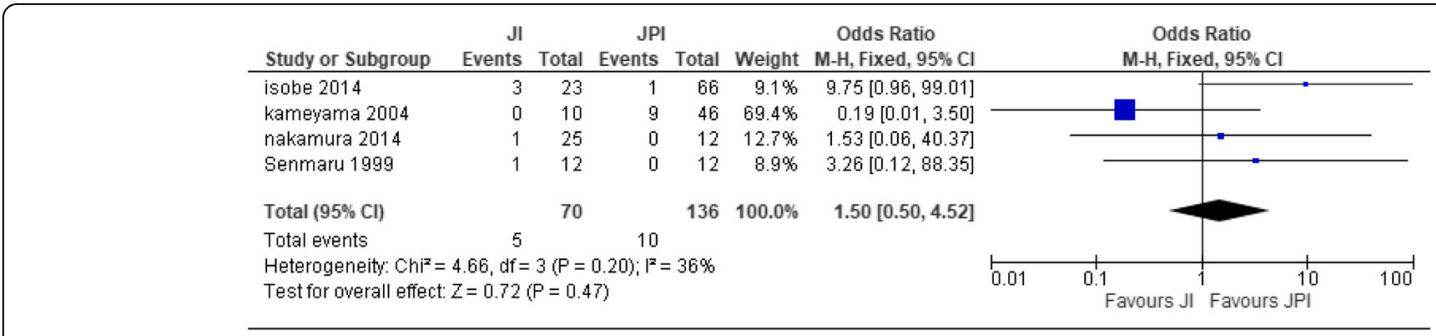

Fig. 4 Forest plot of anastomotic stricture

studies were homogenous; hence, fixed effect was used [heterogeneity: $\mathrm{Chi}^{2}=4.66, \mathrm{df}=3(P=0.20) ; \mathrm{I}^{2}=36 \%$ ]. There was no statistical significance difference between the two methods of reconstruction (test for overall effect: $\mathrm{Z}=0.72(P=0.47))($ Fig. 4$)$.

\section{Operative time}

Three studies $[26,27,41]$ recorded operative time with JI $(n=60)$ patients and JPI $(n=36)$ patients. There was no significant difference between JI and JPI observed. Random effect model was used [Heterogeneity: $\mathrm{Chi}^{2}=$ $\left.5.76, \mathrm{df}=2(P=0.06) ; I^{2}=65 \%\right]$ (test for overall effect: $Z=1.03(P=0.30))($ Fig. 5).

\section{Blood loss}

Two studies [27, 41] were recorded for blood loss with JI $(n=48)$ patients and JPI $(n=24)$ patients. No significant difference was reported. Absolute homogeneity was observed; hence, fixed effect model was used [heterogeneity: $\mathrm{Chi}^{2}=0.31, \mathrm{df}=1(P=0.58) ; I^{2}=0 \%$ ] (test for overall effect: $Z=0.76(P=0.45))$ (Fig. 6).

\section{Hospital length of stay}

Two studie $s[26,27]$ recorded length of hospital stay, with JI $(n=35)$ patients and JPI $(n=24)$ patients. There was a significant difference between the two groups. Patients in the JI group had a decreased length of hospital stay as compared to the JPI group. Heterogeneity was absent; hence, fixed effect model was used. [heterogeneity: $\mathrm{Chi}^{2}=1.34, \mathrm{df}=1(P=0.25) ; \mathrm{I}^{2}=26 \%$.] (test for overall effect: $\mathrm{Z}=2.22(P=0.03)$ ) (Fig. 7).

\section{Meta-analysis result for EG versus J Reflux esophagitis}

Six studies [27, 28, 32, 35, 41, 42] were collected for reflux esophagitis with JI $(\mathrm{n}=146)$ participants and EG ( $\mathrm{n}$ = 241) participants. There was no significant difference between the two groups. Homogeneity was observed between the six studies; thus, fixed effect model was used (heterogeneity: $\mathrm{Chi}^{2}=3.57, \mathrm{df}=5(P=0.61) ; \mathrm{I}^{2}=0 \%$ (test for overall effect: $\mathrm{Z}=1.68(P=0.09))$ (Fig. 8).

\section{Anastomotic stricture}

Four studies [27, 32, 41, 42] recorded anastomotic stricture with JI $(n=98)$ participants and EG $(n=195)$ participants. There was no statistically significant difference between the two groups. Homogeneity was observed; hence, fixed effect was used (heterogeneity: $\mathrm{Chi}^{2}=3.49$, $\mathrm{df}=3(P=0.32) ; \mathrm{I}^{2}=14 \%$ (test for overall effect: $\mathrm{Z}=$ $2.05(P=0.04))$ (Fig. 9).

\section{Anastomotic leakage}

Five studies [27, 28, 32, 41, 42] reported anastomotic leakage with JI $(n=113)$ and EG $(n=174)$. There was no significant difference between the two groups. Homogeneity was observed [heterogeneity: $\mathrm{Chi}^{2}=4.68, \mathrm{df}=3$ $(P=0.20) ; \mathrm{I}^{2}=36 \%$ ] (test for overall effect: $\mathrm{Z}=1.17(P$ $=0.24)$ (Fig. 10).

\section{Operative time}

Six studies [27, 28, 32, 41, 42, 49] collected operative time with JI $(n=160)$ and EG $(n=253)$. A significant difference between the two groups was noted. This study was deemed heterogeneous, as heterogeneity: $\mathrm{Chi}^{2}=$ $31.09, \mathrm{df}=5(P<0.00001) ; \mathrm{I}^{2}=84 \%$. Thence, a random effect model was used (test for overall effect: $Z=32.35$

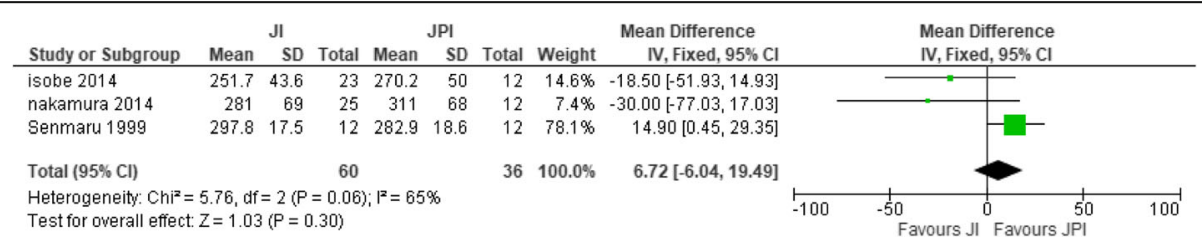

Fig. 5 Forest plot of mean difference in operative time 


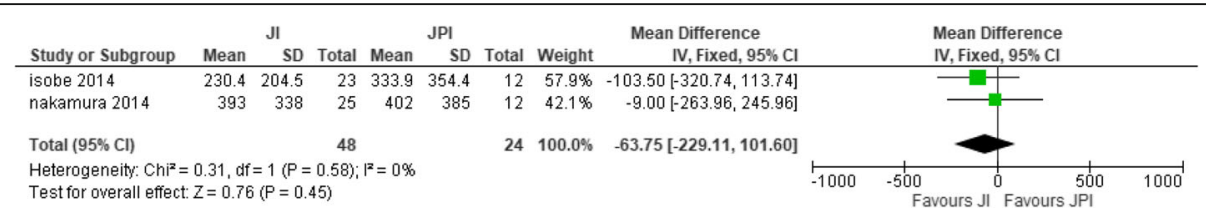

Fig. 6 Forest plot of mean difference blood loss

$(P<0.00001)$. These studies suggested that the EG group had a shorter intraoperative period compared to the JI group (Fig. 11).

\section{Blood loss}

Six studies [27, 28, 32, 35, 41, 42] reported blood loss with JI $(\mathrm{n}=160)$ participants and EG $(\mathrm{n}=253)$ participants. The studies showed a significant difference between the two groups. The EG group reported a decreased amount of blood loss compared to the JI group. Heterogeneity was observed; therefore, fixed effect model was selected (heterogeneity: $\mathrm{Chi}^{2}=11.97, \mathrm{df}$ $=5(P=0.04) ; \mathrm{I}^{2}=58 \%$, test for overall effect: $\mathrm{Z}=5.43$ $(P<0.00001)$ (Fig. 12).

\section{Length of hospital stay}

Four studies [27, 28, 32, 42] collected length of hospital stay with JI $(n=90)$ participants and EG $(n=151)$ participants. There was significant difference between the two groups. The EG group showed a decreased length of hospital stay compared to the JI group. Absolute heterogeneity was estimated; hence, fixed effect was selected (heterogeneity: $\mathrm{Chi}^{2}=1.40, \mathrm{df}=3(P=0.71) ; I^{2}=0 \%$, test for overall effect: $Z=5.04(P<0.00001)$ (Fig. 13).

\section{Publication bias}

The funnel plot on the hospital length of stay between EG and JI is shown in Fig. 14. Because all studies laid inside the 95\% CI limits, no evidence of publication bias was noted. Egger test was performed to provide statistical evidence regarding funnel plot symmetry. Result still did not reveal any evidence of publication bias (heterogeneity: $\left.\mathrm{Chi}^{2}=1.40, \mathrm{df}=3(P=0.71) ; I^{2}=0 \%\right)($ Fig. 15).

\section{Discussion}

The standard surgery preferred for advanced proximal gastric cancer has always favored total gastrectomy, whereas for early-stage proximal gastric cancer, proximal gastrectomy has been the typical surgical solution [29, $50-54]$. The study reviewed and analyzed the postoperative outcomes and effect among 29 studies, and some studies were also used for the meta-analysis. Proximal gastrectomy is a considerable resection procedure for an early stage of proximal gastric cancer providing that a sufficient distal resection margin can be ensured. This has been generally accepted by most GI surgeons [55]. However, in the cases of advanced diseases, it has not reached a consensus for its preference. Moreover, if the rate of reflux esophagitis and anastomotic stricture after proximal gastrectomy can be reduced to that of total gastrectomy, proximal gastrectomy may become an advantageous treatment of choice for proximal EGC [45].

Our review and meta-analysis is based on 5 reconstructive techniques. The totality of the anti-reflux reconstruction methods tested demonstrated excellent ability in preventing reflux. The postoperative effects that were under observance included reflux esophagitis, anastomotic stricture, anastomotic leakage, residual food, operative time, blood loss, and length of hospital stay. Additionally, it was found that the preventive reflux methods within several techniques increased the incidence of residual food, reflux esophagitis, anastomotic stricture, and anastomotic leakage in all the reconstruction methods, but DFT recorded in the studies observed low incidence of complications $8.9 \%, 5.5 \%, 1.4 \%$ and $3.9 \%$ respectively. Individual studies also recorded a low incidence of complications [22, 31, 37, 40, 44]. DFT conducted after proximal gastrectomy seemed to be proven effective to lower the outcome as compared to the procedures of DTR, JPI, JI, and EG. Because of the increased anastomosis numbers and technique complexity, postoperative complications, such as anastomotic leakage, anastomosis stricture, and residual food were shown to increase accordingly. Anastomotic stricture (13.0\%) was frequently seen in the EG group, anastomotic leakage $(18.2 \%)$ was observed in esophagogastrostomy, and

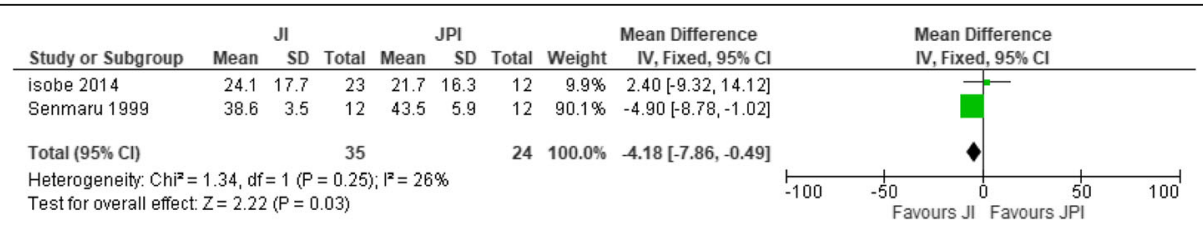

Fig. 7 Forest plot of mean difference hospital length of stay 


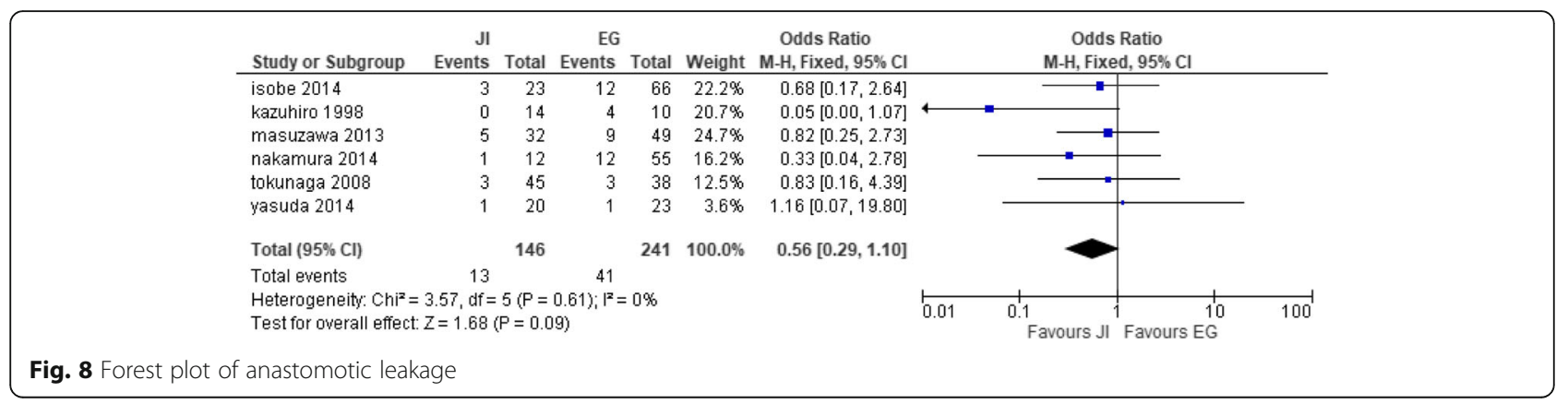

residual food $(100 \%)$ were frequently observed in the jejunal interposition group [32] which were in higher incidence. Other studies have also reported stronger antireflux efficacy [29, 31, 56], whereas others showed a negative result. The operative time in our study was shorter in the EG group (heterogeneity: $\mathrm{Chi}^{2}=31.09$, df $\left.=5(P<0.00001) ; I^{2}=84 \%\right)$, blood loss was less in the EG group (heterogeneity: $\mathrm{Chi}^{2}=11.97, \mathrm{df}=5(P=0.04)$; $I^{2}=58 \%$, and also, there was a decreased length of hospital stay in the EG group (heterogeneity: $\mathrm{Chi}^{2}=1.40, \mathrm{df}$ $\left.=3(P=0.71) ; I^{2}=0 \%\right)$; these results could be attributed to the fact that most surgeries were performed laparoscopically [19, 23, 32, 45, 46].

With the development of clinical research, proximal gastrectomy has been gradually replacing the practice of total gastrectomy in treating early gastric cancer located in the upper third of the stomach. Proximal gastrectomy has maintained comparable oncological radicality to the total gastrectomy and the reservoir capacity of the stomach $[10,18]$ pT1-2 gastric cancer located in the upper third of the stomach has rarely shown any pathological lymph node metastasis at stations \#4d, \#5, and \#6. Although no difference in the long-term survival has been detected between the total and the proximal gastrectomy [49], cardio-esophageal resection and the reserved stomach were shown to significantly increase the risk of gastroesophageal reflux and significantly decrease the postoperative quality of life [18]. PG has a high risk of postoperative gastroesophageal reflux and food stagnation, both of which remarkably decreases the quality of life of patients. Laparoscopic surgery has emerged as an option for the surgical treatment of EGC, including PG.
Laparoscopic reconstruction and the improvements of reconstruction methods in PG to prevent regurgitation of gastric contents and to facilitate their discharge into the duodenum are important issues [32]. Concerning EG reconstruction, Adachi et al. [29] and Shiraishi et al .[57] performed EG with a narrow gastric tube and reported it to be a safe and simple procedure with benefits such as shorter operation time, faster recovery, and lower hospital expenses compared with JI and an equally low incidence of reflux esophagitis despite an end-to-end anastomosis. However, regurgitation when lying down cannot be avoided. Meanwhile, Ichikawa et al. [58] reported that EG was performed in an end-to-side fashion using a narrow gastric tube laparoscopically, resulting in favorable clinical outcomes, along with a low incidence of reflux esophagitis and preservation of the physiological function of the remnant stomach. However, the length of the newly created pseudo-fornix was less than $3 \mathrm{~cm}$, which seemed to be inadequate to function as an angle of his for the prevention of reflux. Related research has also confirmed that PGII improves reflux esophagitis, compared with esophagogastrostomy [16, 35].

Limitations of the techniques were shown to be the incurability of tumors located in the greater curvature and the decreased volume of the stomach, which may alter food intake and nutrition status [29]. So, actual treatment efficacy remains to be determined. There are two major limitations in the present study that was recognized. First, most of the studies on the outcomes of reconstructions for proximal gastrectomy were retrospective case series and non-randomized comparative studies. Also, a downside was that comparisons

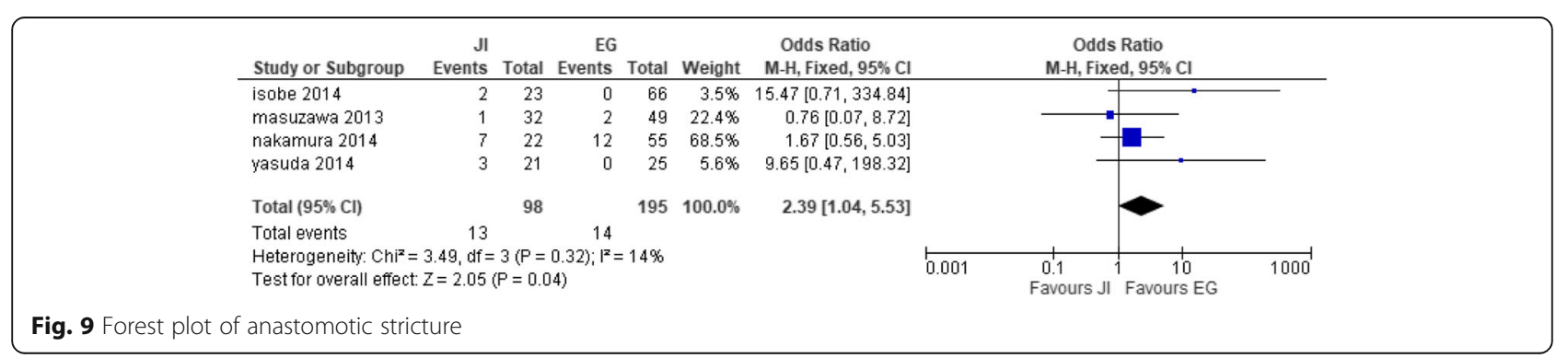




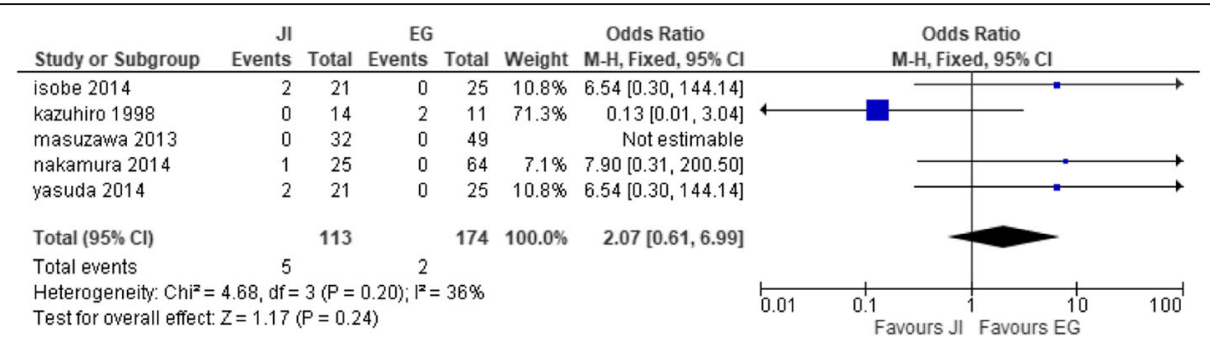

Fig. 10 Forest t plot of anastomotic leakage

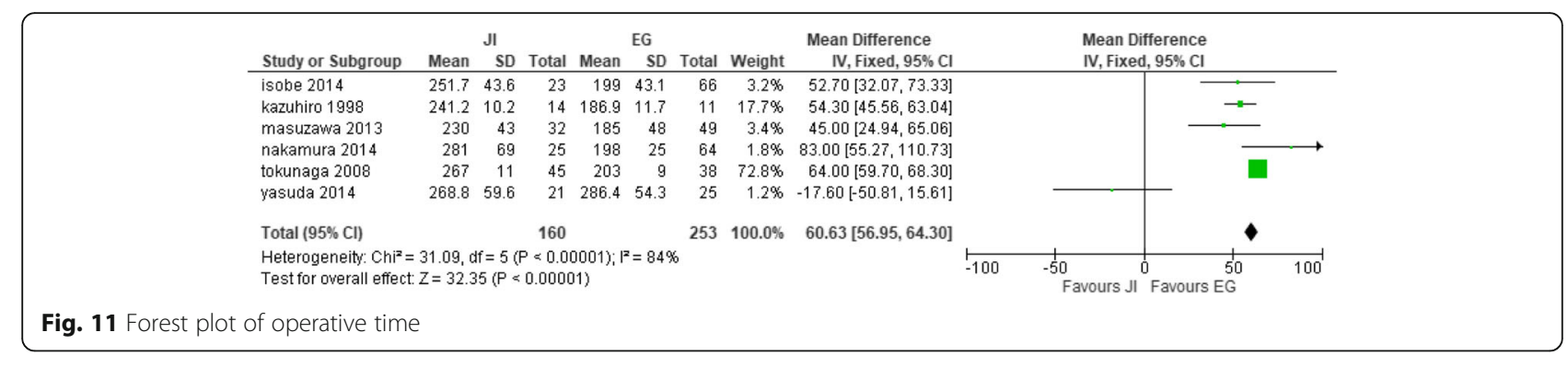

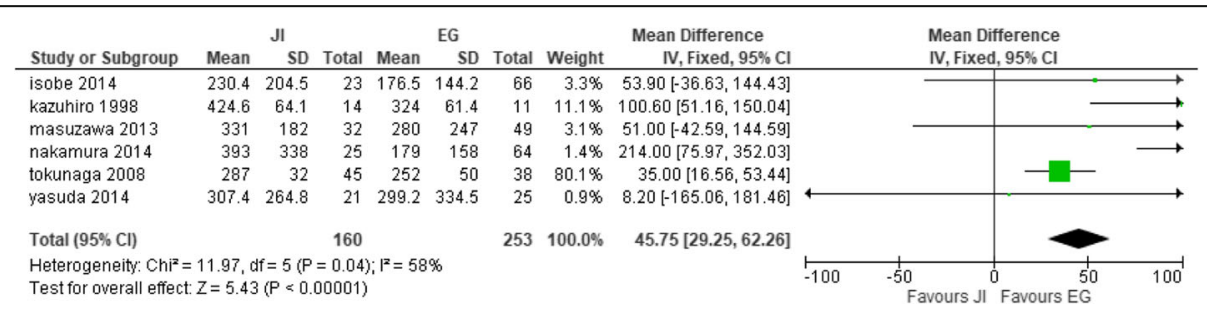

Fig. 12 Forest plot of mean difference blood loss 


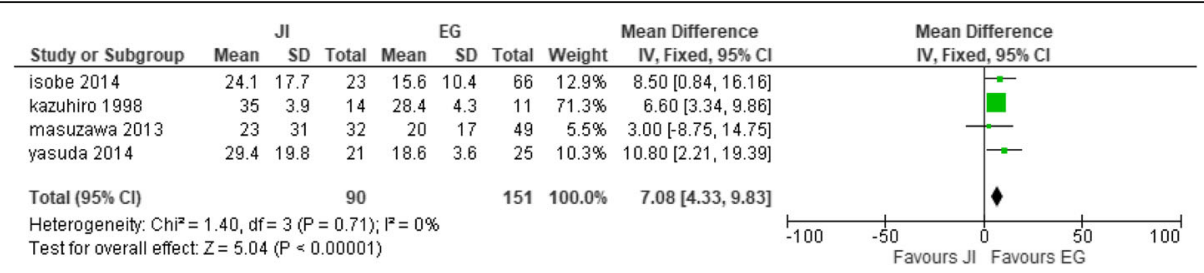

Fig. 13 Forest plot of hospital length of stay.

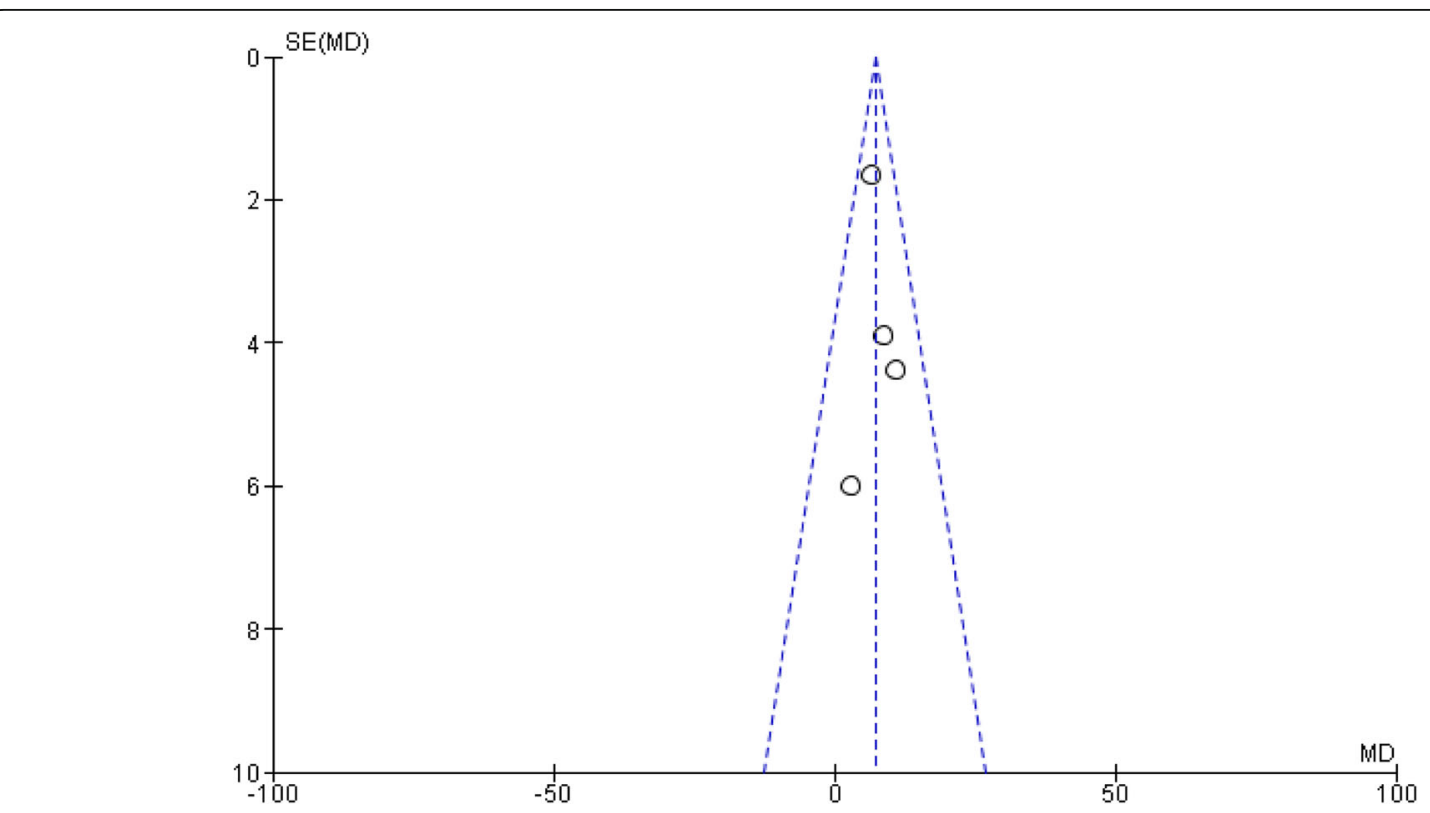

Fig. 14 Funnel plot of hospital length of stay for $\mathrm{Jl}$ and EG
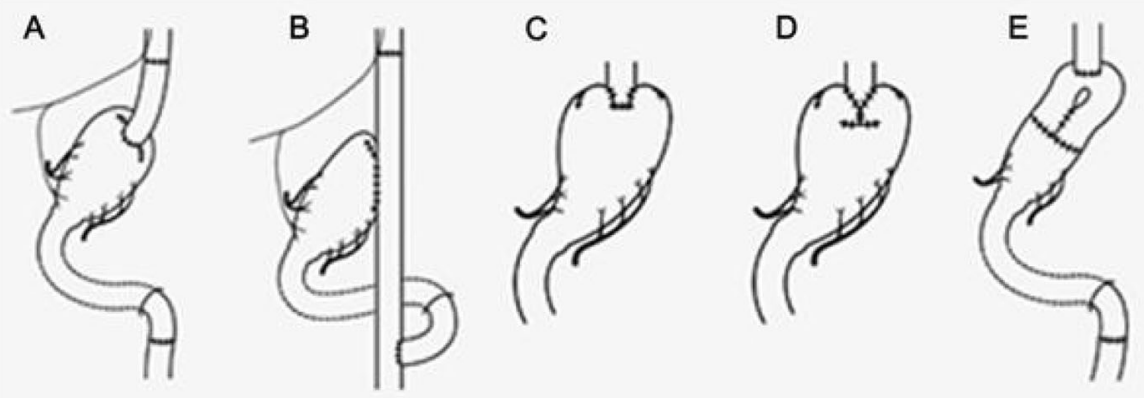

Fig. 15 Reconstruction methods after proximal gastrectomy. a Jejunal interposition. b Double tract method. c Esophagogastrostomy. d Double flap technique. e Jejunal pouch interposition 
between the reconstructions were unavailable. Second, in the included studies, various types of complications and various diagnostic criteria of postoperative reflux esophagitis were adopted. As a result, postoperative complications, including esophageal reflux, were described in general; each type of complication was not described in detail. Given the preliminary stage of this study on the reconstruction following a proximal gastrectomy, it was difficult to estimate the occurrence of each complication and to summarize the presence of reflux esophagitis by each diagnostic criterion. It is important that we must first investigate the general data of the reconstructions.

\section{Conclusion}

In summary, anti-reflux reconstruction methods involved in the studies increased the possible rate of surgical complexity by showing a higher incidence of reflux esophagitis, anastomosis stricture, anastomosis leakage, and residual food. Nevertheless, the double flap technique effectively did decrease the risk of complications after proximal gastrectomy. Also, operative time, blood loss, and hospital length of stay were decreased in the EG group because most surgeries were done laparoscopically. Due to the lack of large randomized studies, optimal anti-reflux methods remain to be unidentified.

\section{Abbreviations}

DTR: Double tract reconstruction; JPI: Jejunal pouch interposition; J: Jejunal interposition; EG: Esophagogastrostomy; DFT: Double flap technique; EGC: Early gastric cancer; PG: Proximal gastrectomy; PGC: Proximal gastrectomy; TG: Total gastrectomy; WMD: Weighted mean difference; $I^{2}$ : I square statistic

\section{Acknowledgements}

We would like to thank professor Zhihong Chen, Dr. Zakari Shaibu, and the entire team in the general surgery department from various hospitals for their input towards the completion of our paper.

\section{Authors' contributions}

Zakari Shaibu, Zhihong Chen, Said Abdulrahman Salim Mzee, Acquah Theophilus, and Isah Adamu Danbala contributed equally to this work and read and approved the final manuscript. They have no conflict of interest or family ties to disclose.

\section{Funding}

This study was supported by the National Science Foundation of China (grant no. 81472334).

\section{Availability of data and materials}

The studies included were retrieved from PubMed, Google scholar, and Medline.

\section{Ethics approval and consent to participate}

Not applicable

\section{Consent for publication}

Not applicable

\section{Competing interests}

The authors declare they have no competing interest.

\section{Author details}

${ }^{1}$ Department of Gastrointestinal Surgery, Affiliated People's Hospital of Jiangsu University, Zhenjiang 212002, Jiangsu, People's Republic of China. ${ }^{2}$ Overseas Education College, Jiangsu university, No 301 xuefu road, Zhenjiang 212013, Jiangsu, People's Republic of China. ${ }^{3}$ Department of Gastrointestinal Surgery, Affiliated Hospital of Jiangsu University, Zhenjiang, Jiangsu, People's Republic of China.

Received: 1 May 2020 Accepted: 29 June 2020

Published online: 16 July 2020

\section{References}

1. Casamayor $\mathrm{M}$, et al. Targeted literature review of the global burden of gastric cancer. Ecancermedicalscience. 2018;12:883.

2. Venerito, M., et al., Gastric cancer: epidemiology, prevention, and therapy. Helicobacter, 2018. 23 Suppl 1: p. e12518.

3. Xin F, et al. Short-term evaluation of immune levels and nutritional values of EN versus PN in gastric cancer: a systematic review and a meta-analysis. World J Surg Oncol. 2019;17(1):114.

4. Bertuccio $P$, et al. Recent patterns in gastric cancer: a global overview. Int J Cancer. 2009;125(3):666-73.

5. Li J, et al. Quality of life after esophagogastrostomy plus gastrojejunostomy reconstruction following proximal gastrectomy: a comparative study of three surgical procedures. Int J Clin Exp Med. 2018;11(9):9791-801.

6. Deans C, et al. Cancer of the gastric cardia is rising in incidence in an Asian population and is associated with adverse outcome. World J Surg. 2011; 35(3):617-24.

7. Zhou Y, et al. A rising trend of gastric cardia cancer in Gansu Province of China. Cancer Lett. 2008;269(1):18-25.

8. Ahn HS, et al. Changes in clinicopathological features and survival after gastrectomy for gastric cancer over a 20-year period. Br J Surg. 2011;98(2): 255-60.

9. Harrison LE, Karpeh MS, Brennan MF. Proximal gastric cancers resected via a transabdominal-only approach. Results and comparisons to distal adenocarcinoma of the stomach. Ann Surg. 1997;225(6):678.

10. Harrison LE, Karpeh MS, Brennan MF. Total gastrectomy is not necessary for proximal gastric cancer. Surgery. 1998:123(2):127-30.

11. Crew KD, Neugut Al. Epidemiology of gastric cancer. World J Gastroenterol: WJG. 2006;12(3):354

12. Martin RC II, et al. Achieving $\mathrm{RO}$ resection for locally advanced gastric cancer: is it worth the risk of multiorgan resection? J Am Coll Surg. 2002; 194(5):568-77.

13. Papachristou D, Fortner J. Adenocarcinoma of the gastric cardia. The choice of gastrectomy. Ann Surg. 1980;192(1):58

14. Hsu, C.-P., et al., Esophageal reflux after total or proximal gastrectomy in patients with adenocarcinoma of the gastric cardia. 1997.

15. Association, J.G.C. Japanese gastric cancer treatment guidelines 2010 (ver. 3). Gastric Cancer. 2011:14(2):113-23.

16. Katai $\mathrm{H}$, et al. Long-term outcome after proximal gastrectomy with jejunal interposition for suspected early cancer in the upper third of the stomach. Br J Surg. 2010;97(4):558-62.

17. Wang $\mathrm{S}$, et al. Reconstruction methods after radical proximal gastrectomy: a systematic review. Medicine. 2018;97:11.

18. Yoo $\mathrm{CH}$, et al. Proximal gastrectomy reconstructed by jejunal pouch interposition for upper third gastric cancer: prospective randomized study. World J Surg. 2005;29(12):1592-9.

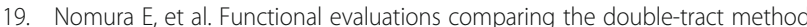
and the jejunal interposition method following laparoscopic proximal gastrectomy for gastric cancer: an investigation including laparoscopic total gastrectomy. Surg Today. 2019;49(1):38-48.

20. Kikuchi S, et al. Results of follow-up endoscopy in patients who underwent proximal gastrectomy with jejunal interposition for gastric cancer. Hepatogastroenterology. 2007;54(73):304-7.

21. KAMEYAMA H, et al. Reconstruction after proximal gastrectomy: comparison with single loop jejunal interposition and jejunal pouch interposition. Nihon Rinsho Geka Gakkai Zasshi (Journal of Japan Surgical Association). 2004; 65(9):2294-8.

22. Kuroda S, et al. Multicenter retrospective study to evaluate the efficacy and safety of the double-flap technique as antireflux esophagogastrostomy after proximal gastrectomy (rD-FLAP Study). Annals of gastroenterological surgery. 2019;3(1):96-103. 
23. Kim DJ, Kim W. Laparoscopy-assisted proximal gastrectomy with double tract anastomosis is beneficial for vitamin B12 and iron absorption. Anticancer Res. 2016;36(9):4753-8.

24. Zhang K, et al. Robot-assisted versus laparoscopy-assisted proximal gastrectomy for early gastric cancer in the upper location: comparison of oncological outcomes, surgical stress, and nutritional status. Cancer Control. 2018;25(1):1073274818765999.

25. Hong J, et al. A novel method of delta-shaped intracorporeal double-tract reconstruction in totally laparoscopic proximal gastrectomy. Surg Endosc. 2016;30(6):2396-403.

26. Senmaru N, et al. Comparative studies between jejunal interposition and jejunal pouch interposition after proximal gastrectomy. The Japanese Journal of Gastroenterological Surgery. 1999;32(10):2309-13.

27. ISOBE T, et al. Reconstruction methods and complications in proximal gastrectomy for gastric cancer, and a comparison with total gastrectomy. The Kurume medical journal. 2014;61(1.2):23-9.

28. Seike $K$, et al. Comparative studies between esophagogastrostomy and jejunal Interposition after proximal gastrectomy for cardiac cancer of the stomach. Nihon Syokakigeka Gakkaizasshi (Jpn J Gastroenterol Surg). 1998; 31:900-7.

29. Adachi Y, et al. Surgical results of proximal gastrectomy for early-stage gastric cancer: jejunal interposition and gastric tube reconstruction. Gastric Cancer. 1999;2(1):40-5.

30. Kondoh Y, et al. Esophagogastrostomy before proximal gastrectomy in patients with early gastric cancers in the upper third of the stomach. Tokai J Exp Clin Med. 2006;31(4):146-9.

31. Omori T, et al. Pure single-port laparoscopic proximal gastrectomy using a novel double-flap technique. ANNALS OF LAPAROSCOPIC AND ENDOSCOPIC SURGERY. 2017;2

32. Yasuda A, et al. A newly modified esophagogastrostomy with a reliable angle of His by placing a gastric tube in the lower mediastinum in laparoscopy-assisted proximal gastrectomy. Gastric Cancer. 2015:18(4):850-8.

33. Nomura $\mathrm{E}$, et al. Functional outcomes by reconstruction technique following laparoscopic proximal gastrectomy for gastric cancer: double tract versus jejunal interposition. World journal of surgical oncology. 2014;12(1): 20

34. Yang K, et al. Laparoscopic proximal gastrectomy with double-tract reconstruction by intracorporeal anastomosis with linear staplers. J Am Coll Surg. 2016;222(5):e39-45.

35. Tokunaga $M$, et al. Endoscopic evaluation of reflux esophagitis after proximal gastrectomy: comparison between esophagogastric anastomosis and jejunal interposition. World J Surg. 2008;32(7):1473-7.

36. Wright C, Cuschieri A. Jejunal interposition for benign esophageal disease. Technical considerations and long-term results. Ann Surg. 1987;205(1):54

37. Saeki Y, et al. Laparoscopic proximal gastrectomy with hinged double flap method using knotless barbed absorbable sutures: a case series. Int J Surg Case Rep. 2018:51:165-9.

38. Tanaka K, et al. Laparoscopic proximal gastrectomy with oblique jejunogastrostomy. Langenbeck's Arch Surg. 2017;402(6):995-1002.

39. Zhao $P$, et al. Proximal gastrectomy with jejunal interposition and TGRY anastomosis for proximal gastric cancer. World J Gastroenterol: WJG. 2014; 20(25):8268

40. Aburatani T, et al. Double-tract reconstruction after laparoscopic proximal gastrectomy using detachable ENDO-PSD. Surg Endosc. 2017;31(11):484856.

41. Nakamura M, et al. Reconstruction after proximal gastrectomy for early gastric cancer in the upper third of the stomach: an analysis of our 13-year experience. Surgery. 2014;156(1):57-63.

42. Masuzawa T, et al. Comparison of perioperative and long-term outcomes of total and proximal gastrectomy for early gastric cancer: a multi-institutional retrospective study. World J Surg. 2014;38(5):1100-6.

43. Takayama Y, et al. Comparison of outcomes of laparoscopy-assisted and open proximal gastrectomy with jejunal interposition for early gastric cancer in the upper third of the stomach: a retrospective observational study. Asian journal of endoscopic surgery. 2018;11(4):329-36.

44. Kano, Y., et al., Laparoscopic proximal gastrectomy with double-flap technique versus laparoscopic subtotal gastrectomy for proximal early gastric cancer. BJS Open, 2019.

45. Ahn S-H, et al. Laparoscopic double-tract proximal gastrectomy for proximal early gastric cancer. Gastric Cancer. 2014;17(3):562-70.
46. Sugiyama M, et al. Laparoscopic proximal gastrectomy maintains body weight and skeletal muscle better than total gastrectomy. World I Surg. 2018;42(10):3270-6.

47. Ohashi $M$, et al. Functional advantages of proximal gastrectomy with jejunal interposition over total gastrectomy with Roux-en-Y esophagojejunostomy for early gastric cancer. World J Surg. 2015;39(11):2726-33.

48. Aburatani T, et al. Double-tract reconstruction after laparoscopic proximal gastrectomy using detachable. ENDO-PSD. 2017;31(11):4848-56.

49. Tokunaga $M$, et al. Effects of reconstruction methods on a patient's quality of life after a proximal gastrectomy: subjective symptoms evaluation using questionnaire survey. Langenbeck's Arch Surg. 2009;394(4):637-41.

50. Sawai K, Takahashi T, Suzuki H. New trends in surgery for gastric cancer in Japan. J Surg Oncol. 1994:56(4):221-6.

51. Kaibara N, et al. Proximal gastrectomy as the surgical procedure of choice for upper gastric carcinoma. J Surg Oncol. 1987;36(2):110-2.

52. Kameyama J, et al. Proximal gastrectomy reconstructed by interposition of a jejunal pouch. The European journal of surgery. 1993;159(9):491-3.

53. Takeshita K, et al. Proximal gastrectomy and jejunal pouch interposition for the treatment of early cancer in the upper third of the stomach: surgical techniques and evaluation of postoperative function. Surgery. 1997;121(3): 278-86

54. Siewert J, et al. Surgical resection for cancer of the cardia. in Seminars in surgical oncology. 1999. Wiley Online Library.

55. Kitamura K, et al. Total gastrectomy for early gastric cancer. J Surg Oncol. 1995;60(2):83-8

56. Uyama I, et al. Laparoscopic side-to-side esophagogastrostomy using a linear stapler after proximal gastrectomy. Gastric Cancer. 2001;4(2):98-102.

57. Shiraishi N, et al. Clinical outcome of proximal versus total gastrectomy for proximal gastric cancer. World J Surg. 2002;26(9):1150-4

58. Ichikawa D, et al. Esophagogastrostomy using a circular stapler in laparoscopy-assisted proximal gastrectomy with an incision in the left abdomen. Langenbeck's Arch Surg. 2012;397(1):57-62.

\section{Publisher's Note}

Springer Nature remains neutral with regard to jurisdictional claims in published maps and institutional affiliations.

Ready to submit your research? Choose BMC and benefit from:

- fast, convenient online submission

- thorough peer review by experienced researchers in your field

- rapid publication on acceptance

- support for research data, including large and complex data types

- gold Open Access which fosters wider collaboration and increased citations

- maximum visibility for your research: over $100 \mathrm{M}$ website views per year

At $\mathrm{BMC}$, research is always in progress.

Learn more biomedcentral.com/submissions 